\title{
Differential proteome analysis of the leaves of lead hyperaccumulator, Rhoeo discolor (L. Her.) Hance
}

Melania M. Enot ${ }^{1 \#}$, Florian Weiland ${ }^{2,3 \#}$, Parul Mittal| ${ }^{2,4}$, Peter Hoffmann ${ }^{2,4}$, Myrna SilleroMahinay ${ }^{5}$, Tara Pukala ${ }^{2,6 *}$

\# These authors contributed equally

${ }^{1}$ Department of Chemistry, Central Mindanao University, Bukidnon, Philippines

${ }^{2}$ Adelaide Proteomics Centre, School of Biological Sciences, University of Adelaide, 5005, Australia

${ }^{3}$ Laboratory of Enzyme, Fermentation and Brewing Technology (EFBT), Department of Microbial and Molecular Systems, Technology Campus Ghent, KU Leuven, Belgium ${ }^{4}$ Future Industries Institute, University of South Australia, Adelaide, 5095, Australia ${ }^{5}$ Department of Chemistry, MSU-Iligan Institute of Technology, Iligan City, Philippines ${ }^{6}$ School of Physical Sciences, University of Adelaide, Adelaide, 5005, Australia

* Correspondence:

Tara Pukala

School of Physical Sciences,

University of Adelaide, Adelaide, 5005, Australia

tara.pukala@adelaide.edu.au

Keywords: metal hyperaccumulators, phytoremediation, protein profiling, leaf proteome, differential protein abundance. 


\begin{abstract}
The present study investigated Rhoeo discolor (L. Her.) Hance for its ability to accumulate Pb, which is of relevance to phytoremediation applications. Based on this analysis, plants were found to accumulate greater than $10 \mathrm{mg} / \mathrm{g}(0.1 \%)$ of dry weight $\mathrm{Pb}$ in the shoots, which classifies the plant a $\mathrm{Pb}$ hyperaccumulator. Further, changes in the leaf proteome profiles in response to $\mathrm{Pb}$ stress were investigated. Wild type plants were subjected to a high concentration of $\mathrm{Pb}\left(\mathrm{NO}_{3}\right)_{2}$ and the levels of $\mathrm{Pb}$ that accumulated in different plant tissues were determined using atomic absorption spectrophotometry. Using 2D-difference gel electrophoresis, 181 protein spots were detected to be differentially abundant in response to $\mathrm{Pb}$ stress and selected spots exhibiting the strongest differential abundance suggested an impairment of the photosynthetic apparatus of the plant under Pb stress. Subsequently, a more extensive, proteome wide analysis utilising label free quantitation further identified a predominant decrease in protein levels, and a significant effect on the nuclear proteome, as well as photosynthesis, carbon fixation and metabolism, providing insight into the $\mathrm{Pb}$ tolerance of this system in a potential phytoremediation context.
\end{abstract}




\section{Introduction}

Lead $(\mathrm{Pb})$ is one of the most toxic and major contaminants found in soil, sediments and water. ${ }^{1}$ It occurs naturally only in small amounts within the earth's crust, ${ }^{2}$ however, common sources for $\mathrm{Pb}$ contamination of the environment derive from frequent use in many industrial processes such as mining and smelting activities, paints containing $\mathrm{Pb}$, gasoline and explosives, as well as the disposal of municipal sewage sludges enriched with $\mathrm{Pb}^{2,3}$ Lead is extremely environmentally persistent, ${ }^{1}$ and usually accumulates in the surface of soils. ${ }^{4}$ Despite regulatory measures and policies adopted in many countries to limit $\mathrm{Pb}$ input into the environment, it continues to be one of the most serious global environmental hazards, especially to plants.

$\mathrm{Pb}$ is a non-essential toxic heavy metal that does not possess biological functions and is able to enter plant tissues through transport systems used by essential micronutrients like $\mathrm{Zn}, \mathrm{Ni}$, $\mathrm{Cu}$ and $\mathrm{Mn}$ which play important roles in different aspects of plant metabolism. ${ }^{5}$ Further, $\mathrm{Pb}$ is easily absorbed and accumulated in different plant tissues and alters the plant's metabolism. ${ }^{6}$ Certain green plant species and genotypes (mostly belonging to the Bassicaceae, Euphorbiaceae, Asteraceae, Lamiaceae and Scrophulariaceae families) can accumulate large quantities of heavy metals, specifically $\mathrm{Pb}$, in their shoot system ${ }^{7}$. Threshold values for metal hyperaccumulation were successfully established, defined according to specific phytotoxicity ${ }^{5}$ and effective transfer of the metal from roots of the plants into their shoots. ${ }^{8}$ Based on these criteria, Pb hyperaccumulators are defined as being able to accumulate greater than $1 \mathrm{mg} / \mathrm{g}(0.1 \%)$ of dry weight $\mathrm{Pb}$ in the shoots ${ }^{5,9,10}$ without suffering phytotoxic damage. ${ }^{5}$ Based on this characteristic, the concept of phytoremediation was developed which uses metal hyperaccumulating plants for the cleansing of contaminated soil and water. ${ }^{11}$

Restoration of polluted areas through phytoextraction requires cultivation of appropriate plant species in situ to allow heavy metal uptake, followed by harvesting of the metalcontaining biomass. This biomass can be treated to decrease its volume/weight and subsequently disposed of as hazardous waste or, if economically viable, utilised for reextraction. Consequently, to be suitable for phytoextraction applications, plant species should 
be capable of high biomass production in the presence of in toxic metal levels and accumulate the metals in easy-to-harvest components. ${ }^{12}$

Many plant species from different families have been found to be effective in removing $\mathrm{Pb}$ from the soil, such as Indian mustard "Brassica juncea", 7 agronomic crops such as pea "Pisum sativum L." and corn "Zea mays", ${ }^{3}$ Sesbania drummondii, ${ }^{13}$ Lespedeza chinensis and Lespedeza davidii. $^{2}$ The plant's ability to tolerate high concentrations of heavy metals depends essentially on mechanisms such as metal transport, sequestration and accumulation. Upon exposure of hyperaccumulator plants to high concentrations of heavy metals, a complex set of mechanisms is activated that regulate the transport of heavy metals into the roots from the soil, loading of absorbed heavy metals into the xylem, and storage and detoxification of heavy metals in the leaves. ${ }^{14}$

Recently, studies on phytoremediation processes have taken advantage of technological advances in proteomics to analyze the profiles of proteins of the complex signalling and metabolic profiles induced by heavy metals. For example, proteomic analysis revealed an increase in relative abundance of 23 proteins in response to lead treatment in Talinum triangulare Jacq. (Willd.) ${ }^{15}$ which were implicated in energy metabolism, reactive oxygen species (ROS) detoxification, cell signalling, primary and secondary metabolism and molecular transport systems.

Unlike in cases of $\mathrm{Zn}$ and $\mathrm{Cd}$ hyperaccumulators, specific mechanisms for $\mathrm{Pb}$ accumulation and tolerance are not well understood. In the present study, Rhoeo discolor (L. Her.) Hance was investigated for the first time for its hyperaccumulating potential for $\mathrm{Pb}$, in combination with proteomic approaches to study $\mathrm{Pb}$-induced responses in the leaves of the plant, to gain better understanding of the molecular basis for metal accumulation and tolerance of $\mathrm{Pb}$ hyperaccumulator plants. The leaf has been chosen for study as it is the target repository part of the plant in the detoxification of toxic heavy metal. ${ }^{16-18}$

\section{Materials and Methods}

\section{Plant growth and heavy metal treatment}


Plant samples of wild-type Rhoeo discolor (L. Her.) Hance of the same height (approx. 6 inches) with 6-8 leaves were extracted from sampling sites roadside in Iligan City, Philippines and thoroughly cleaned to remove soils. Samples were acclimatized for 7 days in hydroponic solution with $\mathrm{pH}$ adjusted to 5.0 with a 16 hour: $8 \mathrm{~h}$ photoperiod regime at $25{ }^{\circ} \mathrm{C}$ during the night and $28^{\circ} \mathrm{C}$ during the day under low intensity light.

The test plants were subjected to $\mathrm{Pb}$ exposure in the form of $\mathrm{Pb}\left(\mathrm{NO}_{3}\right)_{2}$. Treatment commenced with a single application of $1000 \mathrm{ppm} \mathrm{Pb}^{2+}(500 \mathrm{~mL})$, and the volume was maintained by irrigation with hydroponic solution weekly. Following 30 days exposure, 10 biological replicates with 3 technical replicates (total 30 samples) for each of the control and treated plants were harvested with plant parts separated as roots, stems and leaves. Stems and leaves were washed thoroughly with deionized water while root samples were immersed in $10 \mathrm{mM} \mathrm{CaCl}_{2}$ in an ultrasonic bath for $15 \mathrm{~min}$ and then rinsed with deionized water to remove surface bound $\mathrm{Pb}^{2,13}$ The plant tissues were then freeze dried for 3 days followed by oven drying for 10 days at $45^{\circ} \mathrm{C}$ to constant weight. All dried samples were weighed and ground for $\mathrm{Pb}$ analysis.

\section{$\mathrm{Pb}$ metal analysis}

The analytical method for the determination of $\mathrm{Pb}$ employed the sub-sampling process of coning and quartering a representative sample from the freeze-dried plant samples. The material was poured into a conical shape, then flattened into a disc which was divided into quarters; two opposite quarters were discarded, while the other two were combined to constitute a reduced sample. The process was continued until an appropriate sample size remained, thereby reducing sample selection bias from the heterogeneous powder. Approximately $100 \mathrm{mg}$ samples in triplicate (one leaf from each of 3 plants) were digested with a mixture of $5 \mathrm{~mL}$ of $65 \%$ conc. $\mathrm{HNO}_{3}$ and $2 \mathrm{~mL}$ of $30 \% \mathrm{H}_{2} \mathrm{O}_{2}$ in a pressurized oven using an automatic microwave digester (Ethos 1 Microwave Digester, Milestone, USA). The digestion process was set with a temperature of $200^{\circ} \mathrm{C}$ for 45 minutes, applying a pressure of 45 bar, while a $1500 \mathrm{~W}$ microwave power supply was used for heating. Pb levels of the different plant parts were analysed using Flame Atomic Absorption Spectrophotometry 
(Spectra Flame Atomic Absorption Spectrophotometer, Agilent, USA), according to published protocols (Official Methods of Analysis of AOAC International 18th Ed., 2005).

\section{Preparation of protein extracts}

Protein extraction was performed using a trichloroacetic acid (TCA)-acetone protocol allowing the direct precipitation of total proteins ${ }^{19}$ with minor modifications. After 30 days of $\mathrm{Pb}$ treatment, a portion (approx. $0.75 \mathrm{~g} ; 2$ leaves) of medium-age leaf samples from the harvested treated and control plants were cut into small pieces and ground in liquid nitrogen to facilitate cell disruption, transferred to microcentrifuge tubes, and suspended in pre-chilled $\left(-20^{\circ} \mathrm{C}\right) 20 \%$ TCA (LabServ, Ireland) in acetone. The component protein was precipitated overnight at $-20^{\circ} \mathrm{C}$. The TCA/acetone mixture was then centrifuged at $20,000 \mathrm{~g}$ and $-10^{\circ} \mathrm{C}$ for $45 \mathrm{~min}$. The pellets were washed twice with chilled $\left(-20^{\circ} \mathrm{C}\right) 100 \%$ acetone then stored at -20 ${ }^{\circ} \mathrm{C}$ for 1 hour, vortexed thoroughly and centrifuged at $20,000 \mathrm{~g}$ for $45 \mathrm{~min}$. at $10{ }^{\circ} \mathrm{C}$. The protein pellets were stored at $-80^{\circ} \mathrm{C}$ until further use. Three biological replicates from the harvested plants (which were separate to those used for $\mathrm{Pb}$ metal analysis) were subjected to the protein extraction protocol, for both control and treated plants.

For 2D gel electrophoresis, the proteins were solubilized in $500 \mu \mathrm{L}$ TUC4\% buffer (7 M urea (Merck, Germany), 2 M Thiourea (GE Healthcare, UK), 4\% CHAPS (Roche, Switzerland), $30 \mathrm{mM}$ Tris (AmResco, USA), pH 8.5). The suspensions were vortexed thoroughly and remaining DNA was subsequently sheared with ultrasound using a Bioruptor UT200 (Diagenode, Belgium). The samples were then centrifuged at $18,000 \mathrm{~g}$ for 45 minutes at $15^{\circ} \mathrm{C}$ to remove insoluble substances. The supernatants were employed for further analysis. Protein concentration (supplementary data, Table S1) was determined by an EZQ protein quantitation assay (Life Technologies, USA) against an ovalbumin standard curve, performed as described by the manufacturer's protocol.

\section{D-DIGE}

Samples were labelled with fluorescent dyes: G-Dye100, G-Dye200 and G-Dye300 according to the manufacturer's (DyeAgnostics, Germany) minimal labelling protocol. Three biological 
replicates ( $50 \mu \mathrm{g}$ protein each) of the respective experimental group of leaf protein extracts ( $\mathrm{Pb}$ treated vs. control) were labelled, employing a dye-swap ${ }^{20}$ in one of the samples in each group (see supplementary data, Table S2). An internal pooled standard (IPS) was prepared by pooling $25 \mu \mathrm{g}$ of protein from each sample (150 $\mu \mathrm{g}$ of total protein) and labelling with G-100 dye.

Three $24 \mathrm{~cm}$ IPG 3-10NL strips (Bio-Rad, USA) were rehydrated overnight at roomtemperature (RT) using $450 \mu \mathrm{L}$ rehydration buffer containing $6 \mathrm{M}$ urea, $2 \mathrm{M}$ thiourea, $1 \%$ CHAPS, 0.5\% Pharmalytes 3-10 (GE Healthcare, USA) and 200 mM hydroxyethyldisulfide (HED) (Sigma-Aldrich, USA). IEF was performed in the dark on an Ettan IPGphor II (GE Healthcare, UK) at $20^{\circ} \mathrm{C}$ using a 6-step program (Supplementary data, Table S3) with the current limited to $50 \mu \mathrm{A}$ per strip applying anodic cup-loading for sample entry. IEF was terminated after 36,000 Vhs were reached in the final $8,000 \mathrm{~V}$ step and IPG strips were stored overnight at -80 ${ }^{\circ} \mathrm{C}$. The following day, IPG strips were prepared for SDS-PAGE by incubation in $4 \mathrm{~mL}$ equilibration buffer (Serva Electrophoresis $\mathrm{GmbH}$, Germany) with added $6 \mathrm{M}$ urea containing $10 \mathrm{mg} / \mathrm{mL}$ DTT (Roche, Switzerland) for $15 \mathrm{~min}$ on an orbital shaker (40 rpm) in the dark. The equilibration solution was then exchanged with the same equilibration buffer containing 25 $\mathrm{mg} / \mathrm{mL}$ of iodoacetamide (IAA) (GE Healthcare, UK) in place of DTT.

After equilibration, the IPG strips were placed onto 2DGel DALT NF flatbed non-fluorescing pre-cast polyacrylamide gels (Gel Company, USA) ( $T=12.5 \%)$. SDS-PAGE as the second dimension was carried out using a HPE unit (Serva Electrophoresis GmbH, Germany) with the company supplied electrophoresis buffer at $15{ }^{\circ} \mathrm{C}$, according to the manufacturer's protocol. After SDS-PAGE the gels were directly scanned using a Typhoon Trio Image (GE Healthcare, UK) at $100 \mu \mathrm{m}$ resolution. Image analysis was undertaken using DeCyder 2D software (version 7.0, GE Healthcare, UK). Each gel image was processed individually in the Differential in-gel Analysis (DIA) module of DeCyder, prior to exporting to the Biological Variation Analysis (BVA) module for gel-to-gel spot matching and comparative analysis. The data was exported using the XML toolbox module and potentially differentially expressed proteins between the treatment and control group were detected applying a $t$-test with a significance threshold of $\alpha=0.05$, FDR calculations were performed as described previously. ${ }^{21}$ 


\section{Identification of gel spots by LC-MS/MS}

Protein spots were picked using an Ettan Spot Picking robot (GE Healthcare, UK) and subjected to tryptic digestion according to a published procedure. ${ }^{21} \mathrm{LC}-\mathrm{MS} / \mathrm{MS}$ analysis was conducted on an Ultimate 3000 Rapid Separation LC system (Thermo-Fisher Scientific) coupled to an LTQ Orbitrap XL ETD MS instrument (Thermo-Fisher Scientific). One microliter of the peptide sample was pre-concentrated onto a C18 trapping column (Acclaim PepMap100 C18 $75 \mu \mathrm{m} \times$ $20 \mathrm{~mm}$, Thermo-Fisher Scientific) at a flow rate of $5 \mu \mathrm{L} / \mathrm{min}$ in $2 \%$ ACN $0.1 \%$ TFA. Peptides were then separated using a $75 \mu \mathrm{m}$ ID C18 column (Acclaim PepMap100 C18 $75 \mu \mathrm{m} \times 50 \mathrm{~cm}$, Thermo-Fisher Scientific) at a flow rate of $0.3 \mu \mathrm{L} / \mathrm{min}$ using a linear gradient from 2 to $45 \% \mathrm{~B}$ over 80 min ( $\mathrm{A}$ : $2 \%$ aqueous $\mathrm{ACN}$ in $1 \% \mathrm{FA}, \mathrm{B}: 80 \%$ aqueous $\mathrm{ACN}$ in $0.1 \% \mathrm{FA})$. MS scans were acquired in the mass range of 300 to $2000 \mathrm{~m} / \mathrm{z}$ at a resolution of 30000 . The six most intense signals were subjected to CID fragmentation using a dynamic exclusion of $5 \mathrm{~s}$. The fragmentation parameters were minimum signal of $1000 \mathrm{~m} / \mathrm{z}$, isolation width of 3.0, normalized collision energy of 35 , and charge state $\geq 2$.

MS data were submitted to Mascot (V2.3.02, Matrixscience, USA) via Proteome Discoverer Daemon (V1.3, Thermo Scientific) and searched against the SwissProt 2014_02 database. The search parameters were set as: Viridiplantae (34,824 sequences), trypsin digestion, maximum of 1 missed cleavage, fixed carbamidomethylation of cysteine and variable oxidation of methionine, precursor ion mass tolerance of $10 \mathrm{ppm}$, and product ion mass tolerance of 0.8 Da, scoring of peptide sequences was carried out by the Mascot Percolator algorithm. FDR on the peptide level was calculated by MASCOT, selecting the option to search against a decoy database. At an expectation level of 0.05 , the FDR in all analysed samples was $0 \%$, with the exception of Spot 1545 with 1.39\% (one false positive peptide identification in the decoy database).

\section{Label free quantification}

Total protein was extracted from an independent set of wild-type Rhoeo discolor (L. Her.) Hance (separate biological replicates to those used for $\mathrm{Pb}$ metal analysis and proteomics analysis) following the $\mathrm{Pb}$ treatment, extraction, solubilisation and quantification protocol 
described above. Six control and six treated samples were processed according to Wisniewski et al. protocol with slight modifications. ${ }^{22}$ In brief, protein concentration was quantified using an EZQ protein assay (Life Technologies, USA). A total of $50 \mu \mathrm{g}$ of protein extract was used for trypsin digestion. Samples were concentrated onto Vivacon ultrafiltration spin columns (Sartorius Vivacon 500, 10000 MWCO HY, Thermo-Fisher Scientific), which were prewashed with $8 \mathrm{M}$ Urea in $100 \mathrm{mM}$ ammonium bicarbonate (Merck). Samples were reduced with $50 \mathrm{mM}$ DTT (Roche, Switzerland) for 1 hour at $30{ }^{\circ} \mathrm{C}$ with slow agitation. After centrifugation at $14,000 \mathrm{~g}$ for $10 \mathrm{~min}$, a washing step with $8 \mathrm{M}$ urea in $100 \mathrm{mM}$ ammonium bicarbonate buffer was performed. Alkylation was carried out with the addition of $55 \mathrm{mM}$ iodoacetamide (IAA, GE Healthcare) solution (8 $\mathrm{M}$ urea in $100 \mathrm{mM}$ ammonium bicarbonate buffer) and incubated at $25^{\circ} \mathrm{C}$ for $20 \mathrm{~min}$ in the dark. After centrifugation at $14,000 \mathrm{~g}$ for $10 \mathrm{~min}$, proteins were washed with $8 \mathrm{M}$ Urea in $100 \mathrm{mM}$ ammonium bicarbonate then rinsed with $50 \mathrm{mM}$ ammonium bicarbonate. Samples were digested overnight at 37 with sequencing grade trypsin (Promega, USA) at an enzyme to substrate ratio of 1:50. To stop the reaction, peptide samples were acidified to a final concentration of $1 \%$ TFA and dried in a speed vacuum. The dried samples were diluted to $1 \mu \mathrm{g} / \mu \mathrm{L}$ with $3 \%$ acetonitrile in $0.1 \%$ TFA.

$2 \mu \mathrm{l}$ of the peptide solution was injected into the Dionex Ultimate 3000 nano HPLC-system (Thermo Fisher Scientific) coupled to an Impact HD ${ }^{\mathrm{TM}}$ QTOF mass spectrometer (Bruker Daltonics, Bremen, Germany) via an Advance CaptiveSpray source (Bruker Daltonics). Peptide samples were first pre-concentrated onto a C18 trapping column (Acclaim PepMap100 C18 $75 \mu \mathrm{m} \times 20 \mathrm{~mm}$, Thermo-Fisher Scientific) at a flow rate of $5 \mu \mathrm{L} / \mathrm{min}$ in $2 \%(\mathrm{v} / \mathrm{v})$ ACN $0.1 \%$ (v/v) TFA for 10 minutes. Afterwards, separation of the peptides was achieved using a $75 \mu \mathrm{m}$ ID C18 column (Acclaim PepMap100 C18 $75 \mu \mathrm{m} \times 15 \mathrm{~cm}$, Thermo-Fisher Scientific) at flow rate of $300 \mathrm{~nL} / \mathrm{min}$ and using a linear gradient from 5 to $45 \%$ B (A: 2\% (v/v) ACN 0.1\% (v/v) FA, B: $80 \%(\mathrm{v} / \mathrm{v})$ ACN $0.1 \%(\mathrm{v} / \mathrm{v}) \mathrm{FA}$ ) over $60 \mathrm{~min}$, followed by a $10 \mathrm{~min}$ wash with $90 \% \mathrm{~B}$, and a 10 min equilibration with $5 \% \mathrm{~A}$. MS scans were acquired in the mass range of 300 to $2,200 \mathrm{~m} / \mathrm{z}$ in a data-dependent fashion using Bruker's Shotgun Instant Expertise ${ }^{\mathrm{TM}}$ method. This method uses IDAS (intensity dependent acquisition speed) to adapt the speed of acquisition depending on the intensity of precursor ions (fixed cycle time), and RT2 (RealTime Re-Think) to exclude previously selected precursor ions from undergoing re-fragmentation unless the chromatographic peak intensity of the ion has increased by a factor of 5 . Singly charged 
precursor ions were excluded from acquisition. Collision energy ranged from $23 \%$ to $65 \%$ as determined by the $\mathrm{m} / \mathrm{z}$ of the precursor ion. The quality and precision of the data obtained from the proteomic analysis were guaranteed by performing all the experiments in experimental triplicates where $p$-values of $<0.05$ were considered significant.

\section{Data Analysis}

Spectra were analysed using the open source software MaxQuant (version 1.6.10.43) with the Andromeda search engine against the UniProt viridiplantae database. The standard QTOF settings in MaxQuant were used with a mass error tolerance of $20 \mathrm{ppm}$. The variable modification of oxidation of methionine and the fixed modification of carbamidomethyl of cysteines were specified, with the digestion enzyme specified as trypsin. LFQ was activated with minimum ratio count of 2 and allowed match between runs as well as unidentified features. Normalisation of the LC-MS/MS runs was performed based on the least overall proteome variation where majority of the proteins do not change between the samples. The false discovery rate (FDR) was set to $1 \%$ for both proteins and peptides, with a minimum peptide length of 7 amino acids. Only unique and razor peptides (non-unique peptides assigned to the protein group containing the largest number of other peptides) were used when reporting protein identifications and proteins with fewer than two unique peptides were excluded. Further analysis was carried in Perseus software (version 1.6.2.3). Here the data was processed and filtered for potential contaminants, reverse sequences and only identified by sites. Label free intensity was then imputed and transferred by $\log 2(x)$. Only proteins that were present in at least $80 \%$ of one experimental group were considered for further analysis. Statistically significant differences between the two groups was calculated by two sample student's T-test where false discovery rate was set to 0.01 . Enrichment analysis defined by gene ontology (GO) and the database of Kyoto Encyclopedia of Genes and Genomes (KEGG) were performed to categorize and group candidate proteins using ShinyGO (version 0.61$)^{23}$ for the statistically significant proteins from label free quantitation. Gene IDs were search against the best matching species, with a p-value cutoff (FDR) of 0.05 . 
Data has been deposited to the ProteomeXchange Consortium via the PRIDE ${ }^{24}$ partner repository with the dataset identifier PXD003873 and PXD019092. 


\section{Results}

\section{$\mathrm{Pb}$ uptake and accumulation in plants}

To investigate the possible hyperaccumulator properties of Rhoeo discolor (L. Her.) Hance, the plant was first grown under high $\mathrm{Pb}$ conditions. After 30 days growth following $\mathrm{Pb}$ metal exposure (1000 ppm $\mathrm{Pb}^{2+}$ on day one), the plants exhibited tolerance and resistance with minimal toxicity symptoms manifested by wilting and yellowing of the basal leaves and very minimal browning of the roots as assesed by eye.

$\mathrm{Pb}$ content in control and $\mathrm{Pb}$ treated plant tissues was quantified following 30 days growth by atomic absorption spectroscopy. As summarized in Table 1, the roots of the treated wild-type Rhoeo discolor (L. Her.) Hance had the highest level of $\mathrm{Pb}(159.06 \mathrm{mg} / \mathrm{g})$, followed by the stem $(65.53 \mathrm{mg} / \mathrm{g}$ ) while the lowest amount was detected in the in the leaves $(3.25 \mathrm{mg} / \mathrm{g})$. The bioconcentrations of $\mathrm{Pb}$ in the shoot system were based on the dry weights of each stem and leaves samples with the calculated additive value of $68.7795 \mathrm{mg} \mathrm{Pb}$ per g shoot. The results of this study revealed that Rhoeo discolor (L. Her.) Hance displayed high $\mathrm{Pb}$ accumulating capacity, at $6.88 \%$ of the shoot system.

\section{Proteomic analysis of the leaves of Rhoeo discolor (L. Her.) Hance by 2D-DIGE}

Changes in response to $\mathrm{Pb}$ stress in the leaf proteome of Rhoeo discolor (L. Her.) Hance were first broadly examined using 2D-DIGE. Figure 1A shows a representative image of the overlaid G-200/G-300 channels under evaluation. 2129 protein spots on average were resolved from the total proteins of the leaves in each of the 2D gels. 1703 spots were eligible for statistical testing of which 181 spots (Figure $1 \mathrm{~A}$ ) passed the significance threshold of $\alpha=0.05$ (unpaired, two-sided t-test [Supporting Information, Table S4]). The q-value extended false discovery rate was calculated to be $46 \%$ within the $2 \mathrm{D}$ gel spot group passing the significance threshold. Fold change of the protein abundance in each protein spot passing the significance threshold is given by the "average ratio" calculated as group $\mathrm{Pb} / \mathrm{C}$ (where $\mathrm{C}$ and $\mathrm{Pb}$ refer to the control and $\mathrm{Pb}$-treated leaves of Rhoeo discolor (L. Her.) Hance, respectively). A positive ratio means a higher average spot volume in a group " $\mathrm{Pb}$ " which suggests increased protein abundance, while decreased abundance is indicated by a negative ratio. Among the 181 protein spots with 
potentially differentially expressed proteins, 116 spots were decreased in the $\mathrm{Pb}$ treatment group.

Identification of functional proteins that show altered abundance in response to $\mathrm{Pb}$ exposure is a key step in understanding the molecular mechanisms of uptake, accumulation, detoxification and tolerance to the metal for a hyperaccumulator plant such as Rhoeo discolor (L. Her.) Hance. Following 2D-DIGE analysis, five spots exhibiting the lowest p-value in combination with a high fold-change were initially chosen (Spots 1111, 1545, 1738, 2068 and 2096, see Figure 1B) for identification by LC-MS/MS. A protein was considered identified if at least two unique peptides exhibited an expectation value below 0.05 , as calculated by the Mascot Percolator algorithm. Peptide level false-discovery rates can be seen in Supplementary Information (Table S4). The genome of Rhoeo discolor (L. Her.) Hance is not sequenced yet, therefore the MS data was searched against the NCBInr Viridiplantae database, to enable identification of peptides which exhibit conserved sequences between species. The principle proteins identified in the leaves of Rhoeo discolor (L. Her.) Hance are given in Table 2.

As characterized by LC-MS/MS, protein present in spot 1738 was mainly ribulose-1,5bisphosphate carboxylase/oxygenase (RuBisCO), with peptides matched to homologous proteins of various organisms in the database. 2D-DIGE results indicate a decreased abundance of this specific RuBisCO proteoform with a fold change of -2.1 under $\mathrm{Pb}$ stress (Figure 1C and Table S4, Supporting Information). In contrast, for the other spots analysed, LC-MS/MS revealed multiple protein components as result of co-migration in 2D electrophoresis (Table S5 of Supporting Information). While emPAI scores can go some way to estimating relative protein abundance in the spots where co-migration occurs, ${ }^{25}$ this remains a significant limitation of our DIGE analysis. For example, protein abundance in spot 1545 was also found by 2D-DIGE to be decreased under Pb-stress, with a fold change of 2.3 (Table S4 of Supporting Information). MS analysis identified chlorophyll a/b binding protein, along with various additional proteins in this spot (Table S5). Exhibiting a high emPAI value of 1.24, Lhcb1/LHCII type 1- chlorophyll a/b binding protein was chosen for further analysis. Decrease of Lhcb1/LHCII type 1- chlorophyll a/b binding protein abundance under Pb stress was confirmed by western blot analysis (supplementary information, Figure S1). Additionally, 
to exclude influence of other proteoforms of Lhcb1 with the same mass but a different $\mathrm{pl}$, a 2D western-blot was performed, where no additional proteoforms at the molecular mass of the lower band of Lhcb1 were detected by antibody probing (Figure S2). Other than these photosynthetic proteins, further proteins of diverse biological importance to Rhoeo discolor (L. Her.) Hance were identified and quantified by nanoLC-MS/MS for the selected DIGE spots with high ion score and peptide coverage (Table S5 of Supporting Information).

Given the complexity of the DIGE analysis, whereby more than 180 spots were observed to be differentially abundant, many of which seemingly contained multiple proteins, it was not feasible for us to individually characterise and validate the DIGE spots LC-MS and western blotting. Consequently, we turned to label-free quantitative analysis to gain broader insight regarding altered leaf protein expression under $\mathrm{Pb}$ stress.

\section{Label-free quantitation and functional analysis of proteomic changes in Rhoeo discolor (L.}

\section{Her.) Hance Pb stress}

Through a label free-based shotgun quantification approach, a total of 864 protein groups were successfully identified in Rhoeo discolor (L. Her.) Hance leaves. Of these, 62 protein groups (7.2\% of the total proteins) were found to be significantly differentially expressed upon $\mathrm{Pb}$ treatment in the label-free quantitative protein analysis, with all but one of these (Chaperonin 60 subunit alpha 1) showing a decrease in abundance (See Supporting Data File S1 for curated protein list). Notably, for the limited results obtained from DIGE analysis, the significant reduction in RuBis $\mathrm{CO}$ and chlorophyll $\mathrm{a} / \mathrm{b}$ binding protein abundance in $\mathrm{Pb}$ treated leaves was consistent with this label free LC-MS/MS approach with fold changes of -0.9 ( $p$ $=0.05)$ and $-0.9(p=0.03)$ respectively.

Enrichment analysis was then performed to determine if the altered protein set was enriched from genes of a certain pathway or functional category as defined by gene ontology (GO). As can be seen in Table 3, the majority of identified proteins can be assigned to high level GO categories associated with stress and stimuli, consistent with expectation of chemical exposure. Assigning the differentially expressed proteins to functional categories (Figure 2A) 
revealed influence on systems associated with photosynthesis as expected from results of DIGE analysis and previous experimental studies.

Notably, most significant enrichment was found in biological processes and cellular components associated with nucleosome assembly/organization, chromatin assembly/organization, and DNA packaging, structure and interactions (Figure 2A), consistent with the involvement of the nucleus as a primary plant phenotype remodelling response to stress. ${ }^{26}$ Analysis at the molecular function level revealed a number of oxidative and metal ion binding related terms which is also consistent with previous proteomic responses to leadinduced oxidative stress demonstrated in other plant species. ${ }^{15}$ Finally, KEGG pathway analysis showed that the identified proteins were enriched in 7 signal pathways $(p<0.05)$ namely photosynthesis, ribosome, metabolic pathways, arachadoic acid metabolism, glutation metabolism, oxidative phosphorylation and carbon fixation, with effects on photosynthesis being most notable (Figure 2B).

\section{Discussion}

The discovery of hyperaccumulator plant species has revolutionized phytoremediation technology since these plants have an innate capacity to absorb metal at levels 100 -fold greater than those typically measured in shoots of the common nonaccumulator plants. ${ }^{27}$ In this study, Rhoeo discolor (L. Her) Hance was investigated to gain deeper understanding on the effect of $\mathrm{Pb}$ to the plant's metabolism related to accumulation, sequestration, detoxification and tolerance.

Following prolonged exposure to $\mathrm{Pb}$ in the form of $\mathrm{Pb}\left(\mathrm{NO}_{3}\right)_{2}$, measured $\mathrm{Pb}$ levels in the stem and the leaves resulted to $68.7795 \mathrm{mg} \mathrm{Pb}$ per g shoots, which suggests $\mathrm{Pb}$ levels greater than the threshold value of $10 \mathrm{mg} / \mathrm{g}(0.1 \%)$. These values indicate that Rhoeo discolor (L. Her.) Hance is a Pb hyperaccumulator plant according to the classification previously described. 5,10 Furthermore, these results suggest an effective ability of the plant to uptake $\mathrm{Pb}$ into the roots, and transport $\mathrm{Pb}$ from roots to shoots employing the strategy of accumulation and sequestration, thereby demonstrating that the plant has the potential to remediate sites 
contaminated with $\mathrm{Pb}$.

As demonstrated in numerous experimental studies, the toxic effects of $\mathrm{Pb}$ in plants is largely a result of altered photosynthetic processes (Bah et al., 2010). The leaves appear green with $\mathrm{Mg}^{2+}$ ions present due to their key role in the chlorophyll structure essential to the photosynthetic process. Damage to chloroplasts by ion exchange of $\mathrm{Mg}^{2+}$ with $\mathrm{Pb}^{2+}$ effects alterations in the chloroplast's pigment-protein complexes and the Lhcb1/LHCII type Ichlorophyll $\mathrm{a} / \mathrm{b}$ binding protein which renders the antenna complex (the series of light absorbing pigments responsible for transfer of light energy) and its reactions useless for photosynthesis. ${ }^{28}$ Treatment of Rhoeo discolor (L. Her.) Hance with Pb may also potentially disrupt the metalloproteins or enzymatic complexes of the photosystem PSI, an antenna protein complex composed of molecules of chlorophyll and carotenoids, which mediate electron transfer from plastocyanin to ferridoxin. This likely results from displacement of metals in the photosynthesis-related proteins with metal centres, namely cytochromes b6f and ferredoxins that contain Fe in the Fe-S clusters, and copper in plastocyanines, by substituting the other divalent ions. ${ }^{29}$ Specifically, an impairment due to lack of iron which is essential for synthesizing chlorophyll can lead to chlorosis, ${ }^{28}$ as exhibited here in Rhoeo discolor (L. Her.) Hance after long exposure to $\mathrm{Pb}$ metal. Although slight signs of chlorosis on the basal leaves of Rhoeo discolor (L. Her.) Hance was observed after 30 days following Pbtreatment, otherwise the overall physical appearance of the plant was not affected.

Despite the physical appearance, in this study, differential abundance of proteins correlated with reactions in the photosynthetic apparatus was observed, with photosynthetic pathways being most significantly influenced as identified in the KEGG analysis (Figure $2 \mathrm{~B}$ ). The $\mathrm{Pb}$ induced reduction of protein in photosynthesis is manifested (at least in part) by decreased abundance of the photosynthetic proteins RuBisCO and Lhcb1/LHCll type 1- chlorophyll a/b binding protein. This is hypothesized to be due to the competing reactions in the multicomplexed photosynthetic reaction centers. The presence of $\mathrm{Pb}^{2+}$ displaces $\mathrm{Mg}^{2+}$ which might result in inhibition of the biosynthesis of chlorophyll during the light harvesting reaction. The light harvesting complexes of PSII containing chlorophyll lose their function, including the oxygen-evolving complexes, which, containing four manganese atoms stabilizing PSII, are most likely susceptible to displacement by $\mathrm{Pb} .{ }^{30} \mathrm{As}$ a consequence, there 
is a decrease in light absorption by PSII followed by subsequent disruption of electron transfer to PSI.

In addition to effects on the photosynthetic machinery, a number of reports have demonstrated that proteins involved in primary carbon metabolism are decreased in abundance under metal stress. ${ }^{31,32}$ For example, proteins involved in carbon fixation, such as RuBisCO, RuBisCO activase, and carbonic anhydrase were shown to be decreased in abundance in young poplar leaves under $\mathrm{Cd}$ stress, ${ }^{32}$ and a reduction of RuBisCO has also been observed in response to stresses from metals other than $\mathrm{Cd} .{ }^{33}$ However, other studies have demonstrated no significant differences in abundance of these proteins. ${ }^{34}$ While changes to carbon fixation networks were noted as significant from the analysis here, such proteins were amongst the few that were identified as having increased abundance in $\mathrm{Pb}$ stress in this system, by both DIGE and label free methods, suggesting these impacts may vary depending on the plant and metal involved.

The nucleus is the primary organelle involved in plant phenotype remodelling in response to environmental stress since it is involved in transformation of the signal into changes in gene expression. ${ }^{26}$ Enrichment analysis (Table 3 ) highlights that proteins which are significantly different in abundance under $\mathrm{Pb}$ stress primarily correlate with changes in nuclear structure including chromatin organisation and assembly. In addition to being an important regulatory component of gene expression, chromatin is also a focus of research in epigenetics. There is evidence that chromatin is part of the plant somatic memory, meaning gene expression patterns can be stably maintained during propagation after the original trigger has disappeared. ${ }^{35}$ Consequently, the significant changes in nuclear proteins observed here are consistent with transcriptional regulation responses expected in $\mathrm{Pb}$ stress that may impact $\mathrm{Pb}$ uptake and remediation capacities upon subsequent exposures or in propagated progeny.

Overall, to maintain normal growth and development in the presence of external stressors, or at least protect against excess damage, plants need to modulate production of typical stress related proteins with conserveration of energy. Thus, it has been proposed that plants follow a definite pattern of prophylactic and avoidance measures in the early stages of heavy metals stresses, but then opt for survival and acclimatization by switching off a large fraction 
of protein synthesis during prolonged stress. ${ }^{36}$ It is notable that the significant majority of proteins identified in these analyses showed decreases in abundance following Pb treatment, particularly from the label-free quantitative approach. In particular, we observe significant perturbations to networks involved in metabolic pathways, and arachidonic acid/glutation metabolism with a decrease in the abundance of these proteins. This is consistent with an integrated bioinformatics analysis of heavy metal responsive marker genes through Arabidopsis thaliana datasets, which found two thirds of differentially expressed genes were downregulated. ${ }^{37}$ Since exposure to high concentrations of $\mathrm{Pb}$ is known to promote oxidative stress in plants leading to deterioration of proteins through oxidation, ${ }^{38}$ this has also been shown to generally reduce the plant protein pool. ${ }^{39,40}$ It is therefore possible that, despite limited physical evidence at the point of sample collection, the plant was approaching limits of tolerance at these levels of $\mathrm{Pb}$. While evidence for proteins with increasing abundance was found through DIGE analysis, further study is therefore required to validate the changes in abundance of such proteins upon $\mathrm{Pb}$ treatment, and probe their involvement in $\mathrm{Pd}$ uptake and resistance in other tissues of the plant, to ultimately determine $\mathrm{Pb}$ tolerance and utility of Rhoeo discolor (L. Her.) Hance in phytoremediation applications.

\section{Concluding Remarks}

The development of phytoremediation technologies for the plant-based clean-up of contaminated soils is of significant interest as it offers an alternative solution to the serious environmental problems of toxic heavy metal contamination. This promising technology is based on the remarkable abilities of certain plant species to tolerate and accumulate extremely high concentrations of heavy metals. In this study, Rhoeo discolor (L. Her.) Hance was shown to accumulate greater than $10 \mathrm{mg} / \mathrm{g}(0.1 \%(\mathrm{w} / \mathrm{w}))$ of dry weight $\mathrm{Pb}$ on the shoots as assessed by metal analysis. This classifies Rhoeo discolor (L. Her.) Hance as a Pb hyperaccumulator which could potentially be used in phytoremediation.

Notably, the proteome studies revealed that exposure of Rhoeo discolour (L.Her.) Hance to $\mathrm{Pb}$ resulted in significant protein-abundance changes in the leaves despite a minimal physiological response of wilting visible in the basal leaves. These changes largely reflected a 
general decreased protein abundance, possibly suggesting approaches to the limit of tolerance to Pb stress. The study's findings offer baseline data for the identification of the functional proteins in the leaves that could help develop future transgenic plants with enhanced tolerance to $\mathrm{Pb}$ metal and possible potential use in phytoremediation. It can also be a key step to gain deeper understanding of the molecular mechanisms involved in accumulation, sequestration and detoxification processes. Future studies could probe the time course up to and beyond 30 days exposure at varied $\mathrm{Pb}$ levels to investigate $\mathrm{a}$ confirmative trend in proteome changes. 


\section{Conflict of Interest}

None to disclose.

\section{Acknowledgements}

We would like to acknowledge financial support from the Commission on Higher Education Scholarship grant (Ph.D. Sandwich Program) of the Philippine government. We also thank Assoc. Prof. Robert Reid of the School of Biological Sciences, The University of Adelaide, South Australia for providing us with plant growth facilities.

\section{Author's Contribution Statements}

ME assisted in the conception and planning of the research work; wrote the research proposal; conducted the experiments on metal treatment, protein extraction, optimization and validation by western blot; collection of data, data analysis and statistics; and drafted the manuscript. FW has substantial contributions to design, the acquisition, analysis of data for the work, revising it critically for important intellectual content, given final approval of the version to be published. PM performed the label free protein quantification and analysis experiments, also helped in writing the manuscript. TP supported and supervised in planning the research work; oversaw the conduct of the experiments and collection of data; assisted in the data and statistical analysis; and proof-read for critical revision/editing and approval of the manuscript to be published. PH has substantial contributions to the interpretation of data for the work, revising it critically for important intellectual content, given final approval of the version to be published. MSM conceptualized the research work; edited, review and endorsed the research proposal for funding support; supervised the metal uptake experiments and data analysis; endorsement of the manuscript for publication. 


\section{REFERENCES}

[1] Fahr M, Laplaze L, Bendaou N, et al. Effect of lead on root growth. Front Plant Sci. 2013;4175-175.

[2] Zheng L-J, Liu X-M, Lütz-Meindl U, Peer T. Effects of Lead and EDTA-Assisted Lead on Biomass, Lead Uptake and Mineral Nutrients in Lespedeza chinensis and Lespedeza davidii. Water, Air, \& Soil Pollution. 2011;220(1):57-68.

[3] Huang JW, Chen J, Berti WR, Cunningham SD. Phytoremediation of Lead-Contaminated Soils: Role of Synthetic Chelates in Lead Phytoextraction. Environmental Science \& Technology. 1997;31(3):800-805.

[4] Jiang W, Liu D, Hou W. Hyperaccumulation of Lead by Roots, Hypocotyls, and Shoots of Brassica juncea. Biologia Plantarum. 2000;43(4):603-606.

[5] Rascio N, Navari-Izzo F. Heavy metal hyperaccumulating plants: How and why do they do it? And what makes them so interesting? Plant Science. 2011;180(2):169-181.

[6] Cheng S. Effects of heavy metals on plants and resistance mechanisms. A state-of-the-art report with special reference to literature published in Chinese journals. Environ Sci Pollut Res Int. 2003;10(4):256-264.

[7] Alkorta I, Hernández-Allica J, Becerril JM, Amezaga I, Albizu I, Garbisu C. Recent findings on the phytoremediation of soils contaminated with environmentally toxic heavy metals and metalloids such as zinc, cadmium, lead, and arsenic. Reviews in Environmental Science and Biotechnology. 2004;3(1):71-90.

[8] Luo C, Shen Z, Li X. Enhanced phytoextraction of $\mathrm{Cu}, \mathrm{Pb}, \mathrm{Zn}$ and $\mathrm{Cd}$ with EDTA and EDDS. Chemosphere. 2005;59(1):1-11.

[9] Sainger PA, Dhankhar R, Sainger M, Kaushik A, Singh RP. Assessment of heavy metal tolerance in native plant species from soils contaminated with electroplating effluent. Ecotoxicol Environ Saf. 2011;74(8):2284-2291.

[10] Piechalak A, Tomaszewska B, Baralkiewicz D, Malecka A. Accumulation and detoxification of lead ions in legumes. Phytochemistry. 2002;60(2):153-162.

[11] Zhao FJ, Lombi E, McGrath SP. Assessing the potential for zinc and cadmium phytoremediation with the hyperaccumulator Thlaspi caerulescens. Plant and Soil. 2003;249(1):37-43.

[12] Suman J, Uhlik O, Viktorova J, Macek T. Phytoextraction of heavy metals: A promising tool for clean-up of polluted environment? Front Plant Sci. 2018;9(1476).

[13] Sharma NC, Sahi SV, Jain JC. Sesbania drummondii cell cultures: ICP-MS determination of the accumulation of $\mathrm{Pb}$ and $\mathrm{Cu}$. Microchemical Journal. 2005;81(1):163-169.

[14] Clemens S, Palmgren MG, Krämer U. A long way ahead: understanding and engineering plant metal accumulation. Trends Plant Sci. 2002;7(7):309-315.

[15] Kumar A, Majeti NV. Proteomic responses to lead-induced oxidative stress in Talinum triangulare Jacq. (Willd.) roots: identification of key biomarkers related to glutathione metabolisms. Environ Sci Pollut Res Int. 2014;21(14):8750-8764.

[16] Verbruggen $\mathrm{N}$, Hermans $\mathrm{C}$, Schat $\mathrm{H}$. Molecular mechanisms of metal hyperaccumulation in plants. New Phytologist. 2009;181(4):759-776.

[17] Sharma SS, Dietz KJ, Mimura T. Vacuolar compartmentalization as indispensable component of heavy metal detoxification in plants. Plant Cell Environ. 2016;39(5):1112-1126.

[18] Pollard AJ, Powell KD, Harper FA, Smith JAC. The Genetic Basis of Metal Hyperaccumulation in Plants. Critical Reviews in Plant Sciences. 2002;21(6):539-566.

[19] Gorg A, Weiss W, Dunn MJ. Current two-dimensional electrophoresis technology for proteomics. Proteomics. 2004;4(12):3665-3685.

[20] Karp NA, Kreil DP, Lilley KS. Determining a significant change in protein expression with DeCyder ${ }^{\mathrm{TM}}$ during a pair-wise comparison using two-dimensional difference gel electrophoresis. PROTEOMICS. 2004;4(5):1421-1432.

[21] Penno MA, Klingler-Hoffmann M, Brazzatti JA, et al. 2D-DIGE analysis of sera from transgenic mouse models reveals novel candidate protein biomarkers for human gastric cancer. J Proteomics. 2012;7740-58.

[22] Wiśniewski JR, Zougman A, Nagaraj N, Mann M. Universal sample preparation method for proteome analysis. Nat Methods. 2009;6(5):359-362.

[23] Ge SX, Jung D, Yao R. ShinyGO: a graphical gene-set enrichment tool for animals and plants. Bioinformatics. 2020;36(8):2628-2629.

[24] Vizcaíno JA, Csordas A, del-Toro N, et al. 2016 update of the PRIDE database and its related tools. Nucleic Acids Res. 2016;44(D1):D447-456. 
[25] Ishihama $\mathrm{Y}$, Oda $\mathrm{Y}, \mathrm{Tabata} \mathrm{T}$, et al. Exponentially modified protein abundance index (emPAI) for estimation of absolute protein amount in proteomics by the number of sequenced peptides per protein. Mol Cell Proteomics. 2005;4(9):1265-1272.

[26] Kosová K, Vítámvás P, Urban MO, Prášil IT, Renaut J. Plant Abiotic Stress Proteomics: The Major Factors Determining Alterations in Cellular Proteome. Front Plant Sci. 2018;9122-122.

[27] Lasat MM. Phytoextraction of toxic metals: a review of biological mechanisms. J Environ Qual. 2002;31(1):109-120.

[28] Pourrut B, Shahid M, Douay F, Dumat C, Pinelli E. Molecular Mechanisms Involved in Lead Uptake, Toxicity and Detoxification in Higher Plants. In: Heavy Metal Stress in Plants, Berlin, Heidelberg, Springer Berlin Heidelberg; 2013:121-147.

[29] Pourrut B, Shahid M, Dumat C, Winterton P, Pinelli E. Lead uptake, toxicity, and detoxification in plants. Rev Environ Contam Toxicol. 2011;213113-136.

[30] Islam E, Liu D, Li T, et al. Effect of $\mathrm{Pb}$ toxicity on leaf growth, physiology and ultrastructure in the two ecotypes of Elsholtzia argyi. J Hazard Mater. 2008;154(1-3):914-926.

[31] Ahsan N, Renaut J, Komatsu S. Recent developments in the application of proteomics to the analysis of plant responses to heavy metals. PROTEOMICS. 2009;9(10):2602-2621.

[32] Kieffer P, Dommes J, Hoffmann L, Hausman J-F, Renaut J. Quantitative changes in protein expression of cadmium-exposed poplar plants. PROTEOMICS. 2008;8(12):2514-2530.

[33] van Keulen $\mathrm{H}$, Wei R, Cutright TJ. Arsenate-induced expression of a class III chitinase in the dwarf sunflower Helianthus annuus. Environmental and Experimental Botany. 2008;63(1):281-288.

[34] Liu P, Huang R, Hu X, et al. Physiological responses and proteomic changes reveal insights into Stylosanthes response to manganese toxicity. BMC Plant Biol. 2019;19(1):212-212.

[35] Probst AV, Mittelsten Scheid O. Stress-induced structural changes in plant chromatin. Current Opinion in Plant Biology. 2015;278-16.

[36] Thapa G, Sadhukhan A, Panda SK, Sahoo L. Molecular mechanistic model of plant heavy metal tolerance. BioMetals. 2012;25(3):489-505.

[37] Niu C, Jiang M, Li N, et al. Integrated bioinformatics analysis of As, Au, Cd, Pb and Cu heavy metal responsive marker genes through Arabidopsis thaliana GEO datasets. PeerJ. 2019;7e6495-e6495.

[38] Pourrut B, Perchet G, Silvestre J, Cecchi M, Guiresse M, Pinelli E. Potential role of NADPHoxidase in early steps of lead-induced oxidative burst in Vicia faba roots. $J$ Plant Physiol. 2008;165(6):571-579.

[39] Brunet J, Varrault G, Zuily-Fodil Y, Repellin A. Accumulation of lead in the roots of grass pea (Lathyrus sativus $L$.) plants triggers systemic variation in gene expression in the shoots. Chemosphere. 2009;77(8):1113-1120.

[40] Zulfiqar U, Farooq M, Hussain S, et al. Lead toxicity in plants: Impacts and remediation. J Env Manag. 2019;250109557. 


\section{Table and Figure Legends}

Table 1: Pb concentrations of the plant parts of Rhoeo discolor (L. Her.) Hance (mean \pm standard deviation). Values in parentheses refer to equivalent \% $\mathrm{Pb}$ dry weight.

Table 2: Mass spectrometric identification of differentially expressed proteins in the leaves of Rhoeo discolor (L. Her.) Hance following Pb treatment (see Supplementary Table S5 for complete identification list). ${ }^{a}$ theoretical molecular weight in $\mathrm{kDa},{ }^{\mathrm{b}}$ theoretical $\mathrm{pl},{ }^{\mathrm{c}}$ as determined by MASCOT, d sequences with an expectation value below 0.05 , e sequence coverage in percent, ${ }^{f}$ exponentially modified protein abundance index (see Ref ${ }^{25}$ ).

Table 3: Gene ontology enrichment analysis for Rhoeo discolor (L. Her.) Hance proteins with significant differential abundance upon $\mathrm{Pb}$ stress, identified from label free quantitative proteomics.

Figure 1: (A) Differentially abundant proteins ( $p$-value $<0.05$ ) between control and $\mathrm{Pb}$ treatment. (B) Protein Spots picked for identification by MS. (C) Histogram of spots identified by mass spectrometry.

Figure 2: Enrichment analysis for Rhoeo discolor (L. Her.) Hance proteins with significant differential abundance upon $\mathrm{Pb}$ stress, identified from label free quantitative proteomics. (A) Gene ontology enrichment analysis for 20 most significant terms in each category. Dark bars indicate enrichment false discovery rate ( $p$ value, FDR, left axis) and light bars indicate number of genes in the list ( $N$, right axis). (B) Hierarchical clustering tree summarizing the correlation among significant pathways identified in KEGG enrichment analysis. Pathways with many shared genes are clustered together and FDR ( $p$ value) is indicated. 
Table S1. Protein concentration after sample preparation.

Table S2. Experimental design for DIGE labeling of protein samples from Rhoeo discolor (L. Her.) Hance.

Table S3. Isoelectric focusing program.

Table S4. Differentially expressed protein spots in leaves of Rhoeo discolor (L. Her.) Hance following $\mathrm{Pb}$ treatment.

Table S5. Mass spectrometric identification of differentially expressed proteins in the leaves of Rhoeo discolor (L. Her.) Hance following Pb treatment.

\section{Supplementary Methods (Western Blotting)}

Figure S1. Relative abundance of protein from the leaves of Rhoeo discolor (L. Her.) Hance by western blot analysis.

Figure S2. 2D Western-blot of $100 \mu \mathrm{g}$ leaf protein extract. 